\title{
Boosting the Optoelectronic Performance by Regulating Exciton Behaviors in a Porous Semiconductive Metal- Organic Framework
}

Chengyu Liang, ${ }^{\mathrm{a} \dagger}$ Liwei Cheng, ${ }^{\mathrm{a} \dagger}$ Shitong Zhang, ${ }^{\mathrm{a}, \mathrm{c} \dagger}$ Sirui Yang, ${ }^{\mathrm{b}}$ Wei Liu, ${ }^{\mathrm{d}}$ Jian Xie, ${ }^{\mathrm{a}}$ Ming-De Li, ${ }^{\text {b* }}$ Zhifang Chai, ${ }^{a}$ Yaxing Wang, ${ }^{\text {a* }}$ and Shuao Wanga*

a State Key Laboratory of Radiation Medicine and Protection, School for Radiological and interdisciplinary Sciences (RAD-X) and Collaborative Innovation Center of Radiation Medicine of Jiangsu Higher Education Institutions, Soochow University, Suzhou 215123, China

${ }^{b}$ Department of Chemistry and Key Laboratory for Preparation and Application of Ordered Structure Materials of Guangdong Province, Shantou University, Shantou 515063, China

${ }^{c}$ State Key Laboratory of Separation Membranes and Membrane Processes, School of Chemical Engineering and Technology, Tiangong University, Tianjin 300387, China

${ }^{\mathrm{d}}$ School of Environment and Material Engineering, Yantai University, Yantai 264005, China

*Correspoding authors:

Email: shuaowang@suda.edu.cn (Shuao Wang)

Email:yxwang@suda.edu.cn (Yaxing Wang)

Email: mdli@stu.edu.cn (Ming-De Li)

$\dagger$ These authors contributed equally to this work. 


\section{Table of Contents}

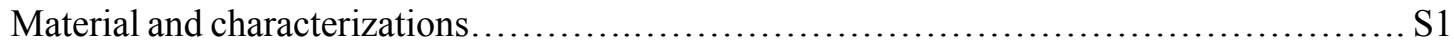

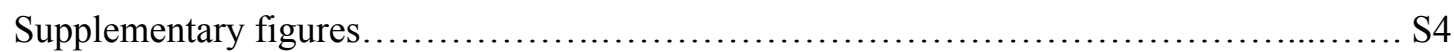

Supplementary Tables..................................... (Table 1) and S15 (Table 2)

Supplementary references............................................................ 16 


\section{Material and characterizations.}

Regents: All reagents were purchased from commercial suppliers without further purification.

Synthesis of TbTATAB. $\mathrm{H}_{3}$ TATAB $(0.05 \mathrm{mmol}, 0.025 \mathrm{~g})$ and $\mathrm{Tb}\left(\mathrm{NO}_{3}\right)_{3} \cdot 6 \mathrm{H}_{2} \mathrm{O}(0.1 \mathrm{mmol}$, $0.0435 \mathrm{~g})$ were dissolved in a mixed solution of deionized water $(2 \mathrm{~mL})$ and DMF $(6 \mathrm{~mL})$. This solution was sealed in a $10 \mathrm{~mL}$ glass vial and heated to $100{ }^{\circ} \mathrm{C}$ for $3 \mathrm{~d}$, and then the reaction was cooled to room temperature. The products were first rinsed with DMF 3 times, rinsed with ethanol and dried in vacuum for $1 \mathrm{~h}$ at $60{ }^{\circ} \mathrm{C}$. The product yield is $79 \%$ based on $\mathrm{Tb}$.

Synthesis of RhB ${ }^{+}$aTbTATAB. TbTATAB (200 mg) was soaked in $80 \mathrm{~mL} 0.1 \mathrm{mmol} / \mathrm{L}$ rhodamine $\mathrm{B}(\mathrm{RhB})$ solution on a shaker for $2 \mathrm{~h}$. After centrifugation, the sample was washed three times with EtOH to remove the $\mathrm{RhB}$ on the surface and then dried in vacuum for $1 \mathrm{~h}$ at $60{ }^{\circ} \mathrm{C}$. As shown in Figure S1, TbTATAB can maintain its crystallinity after adsorption. The adsorbed content was calculated to be $16.42 \mathrm{mg} / \mathrm{g}$ by simulating the adsorption kinetic models, as shown in Figures S16 to S21.

Synthesis of the mechanical mixture. A mechanical mixture of TbTATAB and $\mathrm{RhB}$ was selected as a reference compound. The mixing ratio is guided by the theoretical adsorption value, which is obtained from the adsorption experiment. Typically, $100 \mathrm{mg}$ TbTATAB and $1.64 \mathrm{mg} \mathrm{RhB}$ were mixed well in a mortar and then pressed to a wafer.

Femtosecond Transient Absorption (fs-TA) Experiments. The fs-TA measurement in this work adopts the same experimental setups and methods as before. ${ }^{1}$ In this study, the powder samples were pressed flat on a quartz plate. The sample was excited by a $300 \mathrm{~nm}$ pump beam of the femtosecond regenerative amplified Ti:sapphire laser system.

Fabrication of pelleted wafer-based detectors. Samples were preground in a mortar and then pelleted at $15 \mathrm{MPa}$ in a mold of $3 \mathrm{~mm}$ in diameter to prepare the wafers. The weight of each wafer is controlled at $15 \mathrm{mg}$. Pictures of wafers are shown in Figures S22 and S24. The devices were processed into symmetric structure $\mathrm{Ag} / \mathrm{sample} / \mathrm{Ag}$ and were further embedded in a cathode irradiation configuration to test basic electrical properties.

X-ray detection experiment. Samples for the X-ray detection experiment were preground in a mortar and then pelleted at $15 \mathrm{MPa}$ in a mold $3 \mathrm{~mm}$ in diameter to prepare the wafers. The weight of each wafer was controlled at $15 \mathrm{mg}$, and the thickness was considered separately (approximately $1.3 \mathrm{~mm}$ ). Pictures of wafers are shown in Figures S21 and S22 in the supporting information. The devices were processed into symmetric structure $\mathrm{Ag} / \mathrm{sample} / \mathrm{Ag}$ and were further embedded in a cathode irradiation configuration to test basic electrical properties. X-ray detection and irradiation experiments were performed with the use of a tungsten anode radiation device (RAD SOURCE RS 2000X). The dose rate can be switched by changing the tube current, and it was calibrated in the range of $0.883 \mu \mathrm{Gy}_{\text {air }} \cdot \mathrm{s}^{-1}$ to $17.52 \mathrm{mGy}_{\text {air }} \cdot \mathrm{s}^{-1}$. To minimize the effect of ionization of particles in air, we inserted the irradiation configuration into the electromagnetic shielding loading platform. It is worth mentioning that shielding will not affect 
the X-ray-induced photocurrent. The entire test unit is placed under the X-ray generator. The bias voltages were applied, and then the X-ray-generated photocurrent was recorded by a Keithley 6517B electrometer/high resistance meter.

Physical characterizations. PXRD data were collected from 3 to $50^{\circ}$ on a Bruker D8 advance diffractometer with $\mathrm{Cu} \mathrm{K} \alpha$ radiation $(\lambda=1.54056 \AA)$ with a Lynxeye one-dimensional detector. The single crystal solid-state UV-vis absorption spectrum and emission spectrum were recorded using a Craic Technologies microspectrophotometer. The liquid-phase UV-Vis absorption spectra were recorded using a Shimadzu UV-3600 spectrophotometer. The UPS measurements were collected by Thermo Scientific Escalab 250Xi with the He I ( hv $=21.2 \mathrm{eV}$ ) source. Fourier transform infrared spectroscopy (FTIR) spectra were collected with powder samples by using a Thermo Scientific Nicolet iS50 FT-IR in the range of $400 \sim 4000 \mathrm{~cm}^{-1}$. Scanning electron microscopy/energy-dispersive spectroscopy (SEM/EDS) data were obtained on an FEI Quanta 200FEG. The elemental mapping data were obtained by using an EDAX-PV 9100 instrument. Thermogravimetric analysis was performed by using a NETZSCH STA 449 F3 from 30 to $900{ }^{\circ} \mathrm{C}$ under nitrogen flow with a step of $10{ }^{\circ} \mathrm{C} / \mathrm{min}$.

\section{Characterization of the exciton binding energy $\left(E_{b}\right)$.}

The $E_{b}$ is estimated from the temperature-dependent photoluminescence spectra by fitting the Arrhenius equation:

$$
I(T)=\frac{I_{0}}{1+A e^{\frac{-E_{b}}{k_{B} T}}}
$$

where $\mathrm{I}_{0}$ is the PL intensity at $0 \mathrm{~K}, \mathrm{E}_{\mathrm{b}}$ is the exciton binding energy and $\mathrm{k}_{\mathrm{B}}$ is the Boltzmann constant.

\section{Characterization of the mobility-lifetime product $(\mu \tau)$.}

The $\mu \tau$ product can be derived from the bias-dependent photocurrent curve by employing a modified Hecht equation:

$$
I=\frac{I_{0} \mu \tau V}{L^{2}}\left[1-\exp \left(\frac{-L^{2}}{\mu \tau V}\right)\right]
$$

where $\mathrm{L}$ is the thickness of the wafer detector, $\mathrm{I}_{0}$ is the saturated photocurrent, and $\mathrm{V}$ is the applied bias.

\section{Adsorption data fitting by pseudosecond-order kinetic models.}

The adsorption data were fitted by a pseudosecond-order kinetic model, described as

$\frac{d q_{t}}{d t}=k_{2}\left(q_{e}-q_{t}\right)^{2}$

where $\mathrm{q}_{\mathrm{e}}$ and $\mathrm{q}_{\mathrm{t}}$ are the amount of $\mathrm{RhB}^{+}$adsorbed on the adsorbents at equilibrium and at time $\mathrm{t}$ (min), respectively, and $\mathrm{k}_{2}$ is the rate constant of pseudosecond-order biosorption.

When considering the integration of boundary conditions in which $t=0$ and $q_{t}=0$ to $t=t$ and $\mathrm{q}_{\mathrm{t}}=\mathrm{q}_{\mathrm{e}}$, Eq. (1) can linearly become 
$\frac{t}{q_{t}}=\frac{1}{k_{2} q_{e}^{2}}+\frac{t}{q_{e}}$

In addition, a standard curve of absorbance of $\mathrm{RhB}$ solution was established from $\mathrm{c}_{1}=0.0025$ $\mathrm{mmol} / \mathrm{L}$ to $\mathrm{c}_{5}=0.0125 \mathrm{mmol} / \mathrm{L}$. The curves satisfy the pseudosecond-order kinetic model of chemical adsorption. This type of adsorption involves electron sharing or electron transfer between the host and guest.

\section{The energy-level diagram analysis.}

The UPS spectra were employed to calculate the energy-level diagram for different samples (Figure S23). The HOMO energy is determined using the incident photon energy, $h v=21.2$ $\mathrm{eV}, \mathrm{E}_{\text {cutoff }}$, and $\mathrm{E}_{\text {onset, }}$ which can be described as

$$
E_{\text {HOMO }}=h v-\left(E_{\text {cutoff }}-E_{\text {onset }}\right)
$$

The LUMO energy was calculated using the HOMO levels and the energy gaps $\left(\mathrm{E}_{\mathrm{g}}\right)$.

\section{Calculation methods:}

The initial position and orientation of the cationic $\mathrm{RhB}^{+}$in the pores of the MOF were predicted by molecular dynamics (MD) simulations using the GROMACS software package (version 4.6.6) with a universal force field. ${ }^{2}$ The atomic charges were described by the extended charge equilibration (EQeq) method. ${ }^{3}$ The temperature was maintained at $300 \mathrm{~K}$ by the $v$ rescale thermostat method. ${ }^{4}$ The pressure was coupled at $0.1 \mathrm{MPa}$ using the semiisotropic Parrinello-Rahman barostat method. ${ }^{5}$ The particle mesh Ewald (PME) method ${ }^{6}$ was employed to calculate the long-range electrostatic interactions, and a cutoff distance of $1.5 \mathrm{~nm}$ was used to treat the van der Waals ( $\mathrm{vdW}$ ) interactions. The periodic boundary condition was applied on all systems. The LINCS algorithm ${ }^{7}$ was adopted to maintain constant bonds at their equilibrium values. $\mathrm{RhB}^{+}$was first randomly placed in the $1 \mathrm{D}$ channel of the MOF by avoiding too close a distance to any atoms of the host framework, and the position of the atoms in the MOF was frozen. The minimization of the model was then performed using the steepest descent minimization algorithm. The total time of each data-production simulation was $50 \mathrm{~ns}$, which was conducted in the $N P T$ ensemble. The time step was $2.0 \mathrm{fs}$, and simulation data were collected every $1 \mathrm{ps}$. Three independent trajectories were generated to enhance sampling. The MD-simulated position and orientation of $\mathrm{RhB}^{+}$in the MOF were adopted as the starting points for related DFT geometry optimization.

The initial structure of the MOF is built according to the experimental data with a threedimensional size of $28.65 \times 22.83 \times 15.16 \AA^{3}$ in the a-, b- and c- directions, which contains 8 $\mathrm{Tb}$ atoms and 8 ligands. The structures of the $\mathrm{MOF}, \mathrm{RhB}^{+}$and $\mathrm{RhB}^{+} @ \mathrm{MOF}$ systems (containing one $\mathrm{RhB}^{+}$) were further optimized using the density functional theory (DFT) method within the CASTEP program. ${ }^{8}$ The Perdew-Burke-Ernzerhof (PBE) generalized gradient approach (GGA) $)^{9-11}$ was employed to describe the exchange-correlation potential. Brillouin zone sampling was performed using a $1 \times 1 \times 1 k$-point mesh. Ultrasoft pseudopotentials was adopted in this study. The SCF convergence criterion was set to $1.5 \times 10^{-6} \mathrm{eV} / \mathrm{atom}$, and the convergence criterion of structure optimization was $1.5 \times 10^{-5} \mathrm{eV} / \mathrm{atom}$. The Kohn-Sham orbitals were expanded in a plane wave basis set using a cutoff energy of $340.0 \mathrm{eV}$. The Bader charge analysis was performed according to the method developed by the Henkelman Group. ${ }^{12}$ 
The interaction between the $\mathrm{Cl}$ anions and the deprotonated ligand was simulated using the DFT method with Gaussian 09 software. ${ }^{13}$ The initial model of the ligand was created according to the local structure (removing the $\mathrm{Tb}$ and $\mathrm{O}$ atoms) from the DFT-optimized geometry of the MOF, and each terminated $\mathrm{C}$ (saturated with one $\mathrm{H}$ atom) was kept fixed during the subsequent optimization calculations. Geometries were optimized using the M06-2x method ${ }^{14}$ with a $6-311 \mathrm{G}(\mathrm{d}, \mathrm{p})$ basis set. ${ }^{15}$ The description of van der Waals interactions was improved using Grimme's empirical dispersion (GD3) correction. ${ }^{16}$

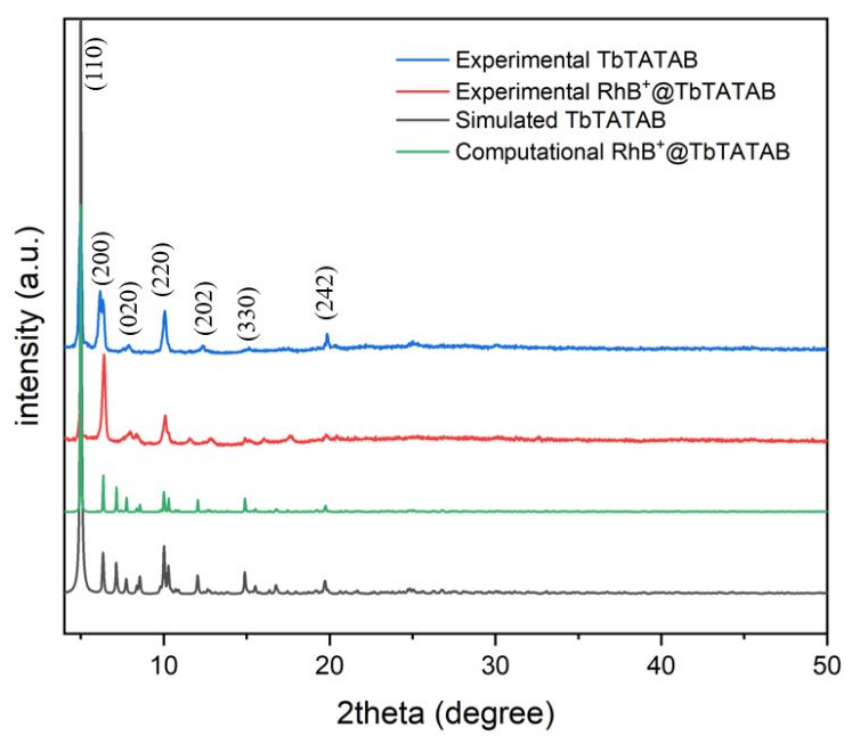

Figure S1. Experimental PXRD data for TbTATAB and $\mathrm{RhB}^{+} @$ TbTATAB and simulated PXRD data from the computational model of RhB ${ }^{+} @$ TbTATAB.
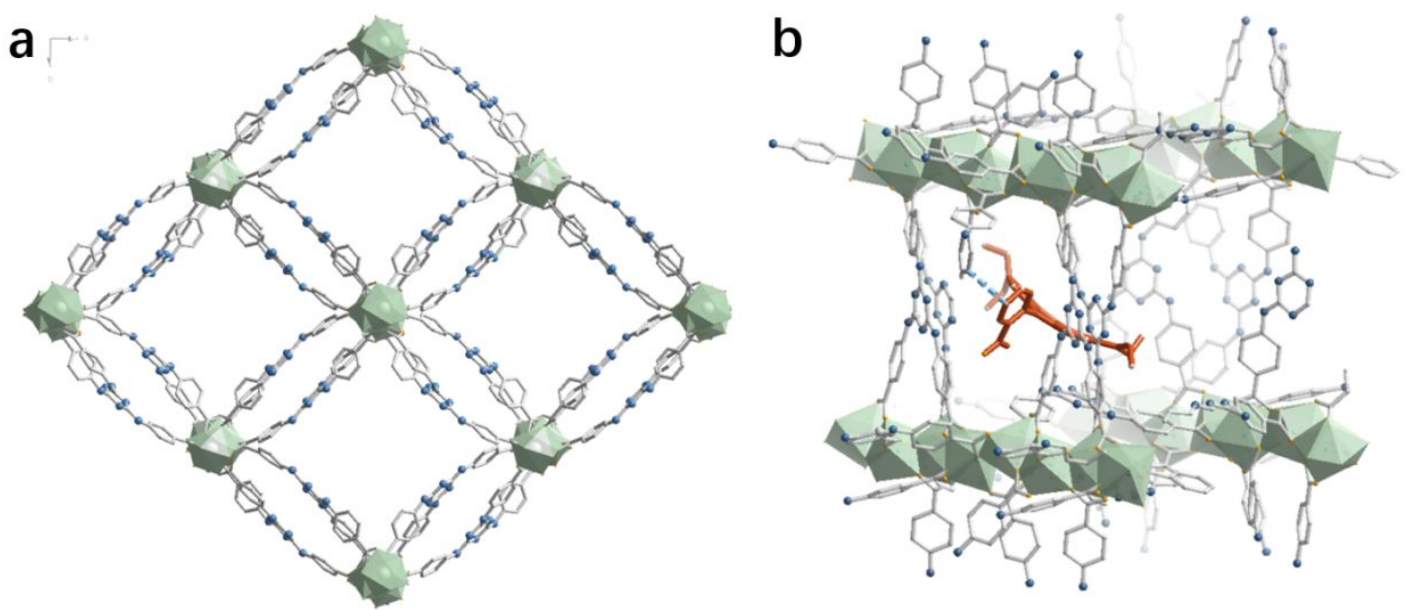

Figure S2. Illustration of the crystal structures of a) TbTATAB and b) RhB ${ }^{+} @ T_{\text {TbTATAB. }}$ 


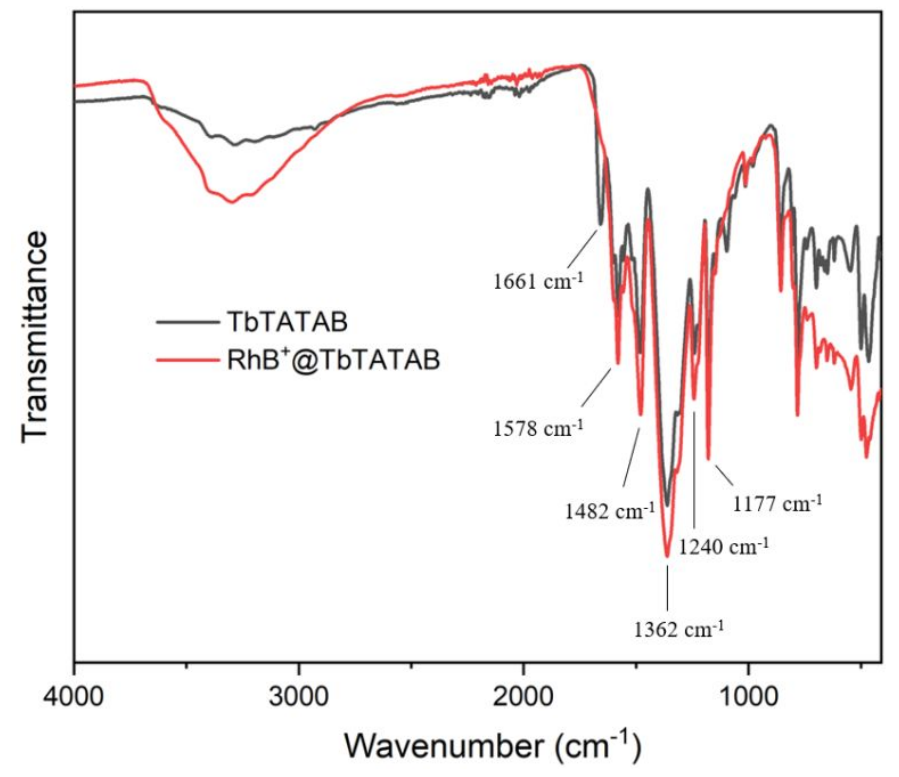

Figure S3. The IR spectra of TbTATAB and RhB ${ }^{+} @$ TbTATAB.

Table S1. Detailed attributes of the IR data.

\begin{tabular}{cc}
\hline Vibration & Wavenumber \\
\hline $\mathrm{V}_{\mathrm{Tb}-\mathrm{O}}$ & $450-706 \mathrm{~cm}^{-1}$ \\
$\mathrm{~V}_{\mathrm{C}-\mathrm{N}}$ & $1176,1240 \mathrm{~cm}^{-1}$ \\
$\mathrm{~V}_{\mathrm{C}=\mathrm{O}, \mathrm{C}=\mathrm{N}}$ & $1482 \mathrm{~cm}^{-1}$ \\
$\mathrm{~V}_{\text {Aromatic ring }}$ & $1578 \mathrm{~cm}^{-1}$ \\
$\mathrm{~V}_{\mathrm{C}=\mathrm{O}(\mathrm{DMF})}$ & $1661 \mathrm{~cm}^{-1}$ \\
\hline
\end{tabular}
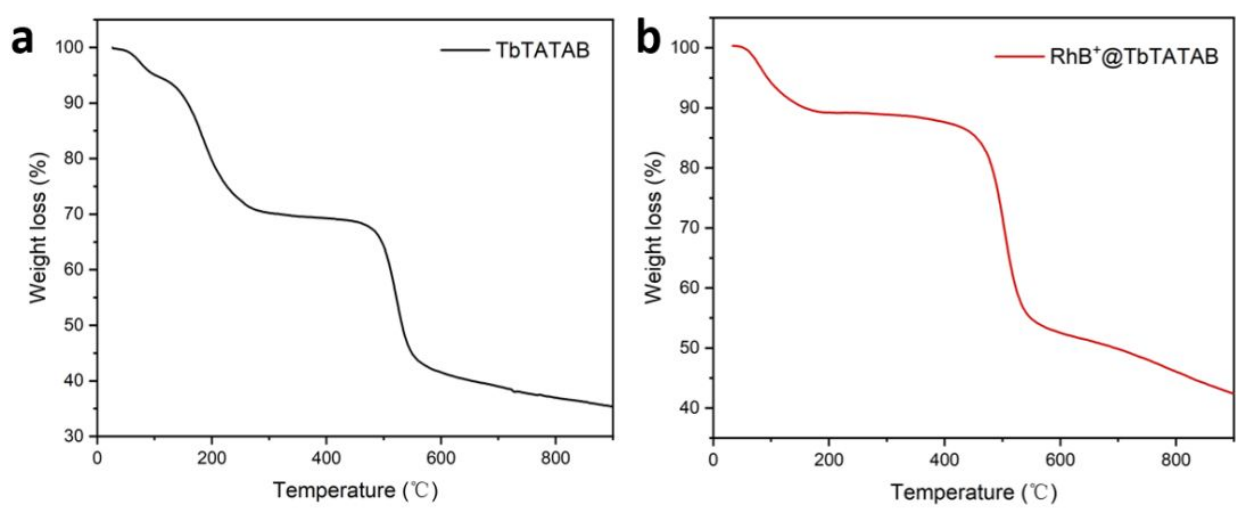

Figure S4. The TGA curves of (a) TbTATAB and (b)RhB ${ }^{+} @$ TbTATAB. The assigned formula of MOF $\left(\mathrm{Tb}_{2} \mathrm{~L}_{2} \bullet 4 \mathrm{H}_{2} \mathrm{O} \bullet 6 \mathrm{DMF}, \mathrm{L}=\mathrm{TATAB}^{3-}\right)$ can be derived from the TGA data. We can see two stages of weight loss in the range of $30 \sim 100{ }^{\circ} \mathrm{C}$ and $100 \sim 270{ }^{\circ} \mathrm{C}$ (Figure S3a). The weight loss in the range of $30 \sim 100{ }^{\circ} \mathrm{C}$ is attributed to the loss of four free water molecules per formula 
of TbTATAB, in which the experimental weight loss of $4.62 \%$ is close to the calculated result from formula $(4.01 \%)$. In the range of $100 \sim 270{ }^{\circ} \mathrm{C}$, the weight loss of $24.03 \%$ corresponds to the loss of six free DMF molecules (calculated result is $24.42 \%$ ).

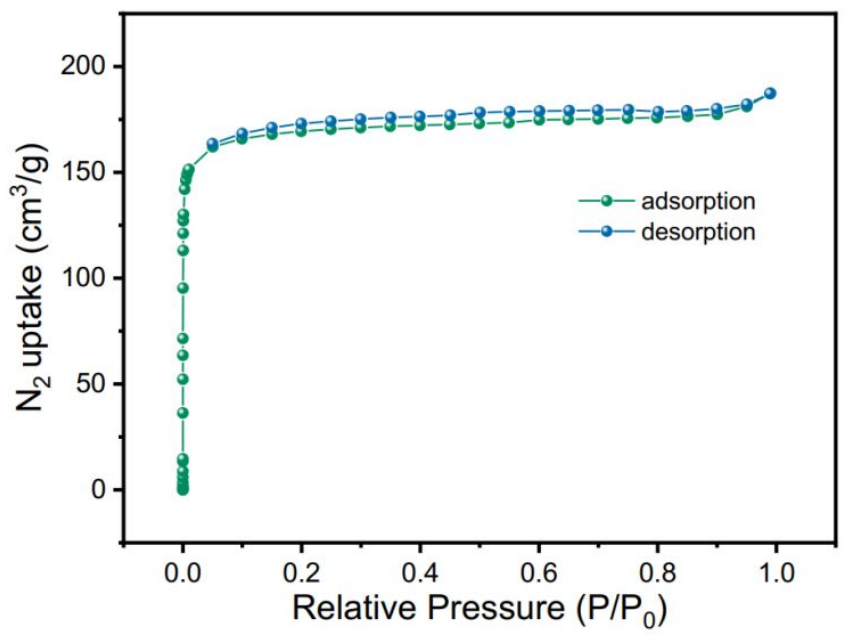

Figure S5. $\mathrm{N}_{2}$ sorption isotherm for TbTATAB at $77 \mathrm{~K}$. The surface areas derived from $\mathrm{N}_{2}$ sorption data are $663.4 \mathrm{~cm}^{2} / \mathrm{g}(\mathrm{BET})$ and $670.4 \mathrm{~cm}^{2} / \mathrm{g}$ (Langmuir).
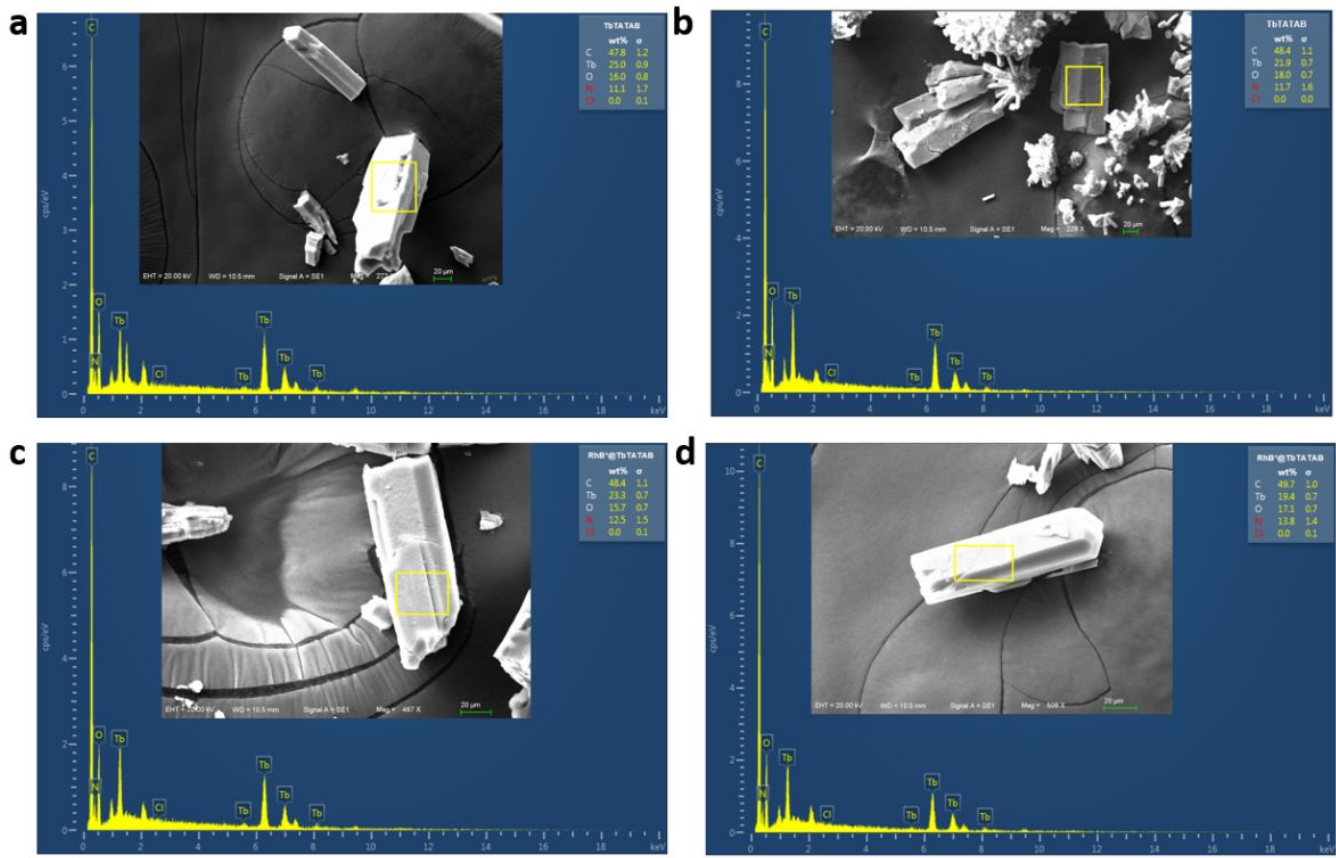

Figure S6. EDS analysis and SEM images (insert) of (a and b) TbTATAB and (c and d)

RhB $@$ @TbTATAB crystals 


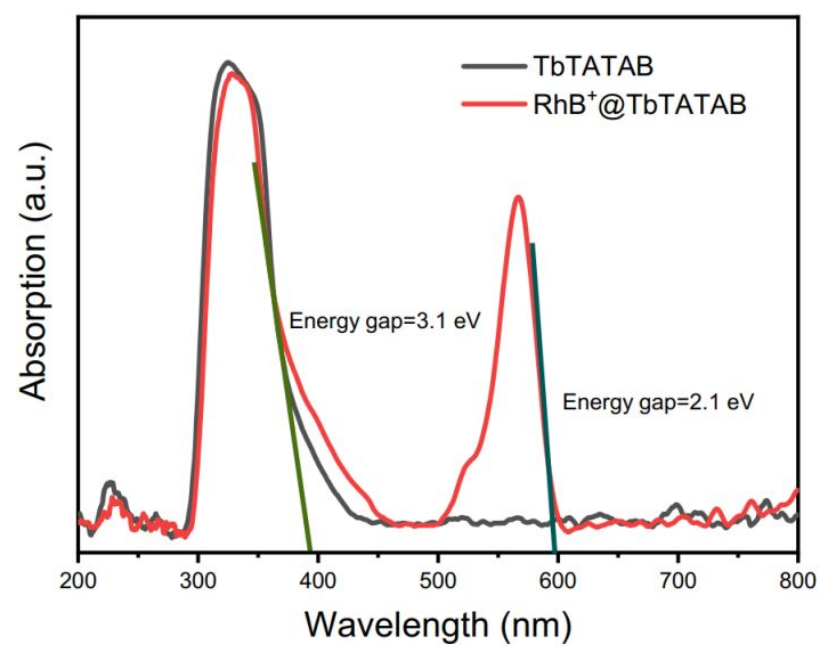

Figure S7. UV-vis absorption spectra for TbTATAB and RhB ${ }^{+} @$ TbTATAB. The energy gaps can be analyzed according to absorbance versus optical energy plots. The calculated energy gap values for TbTATAB and $\mathrm{RhB}^{+} @$ TbTATAB are $3.1 \mathrm{eV}$ and $2.1 \mathrm{eV}$, respectively.

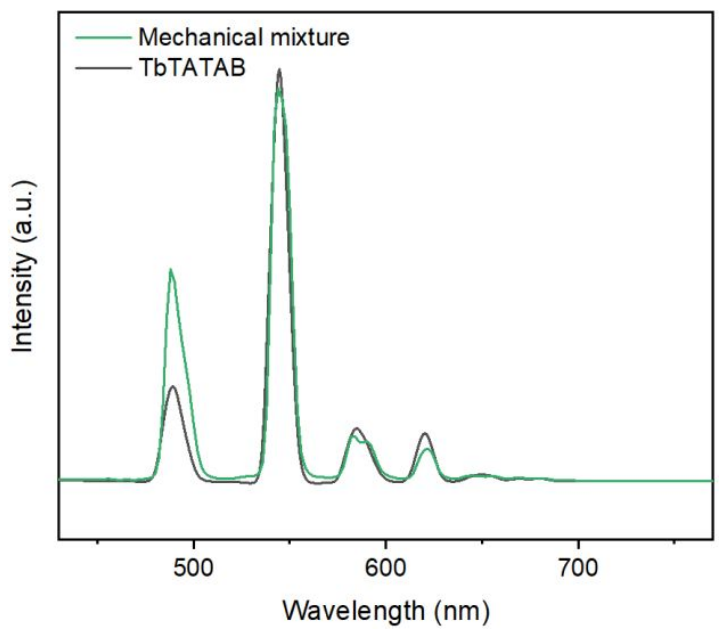

Figure S8. Emission spectra of TbTATAB and a mechanical mixture of TbTATAB and RhB.

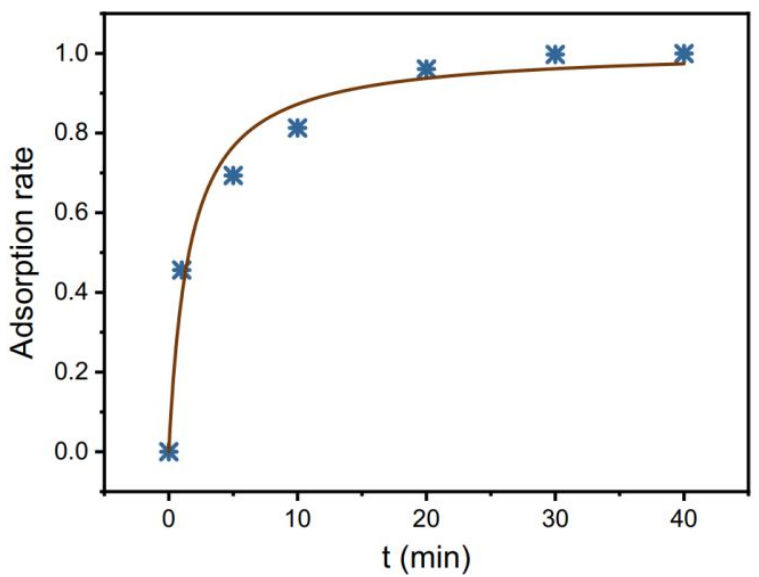

Figure S9. Adsorption rate of $\mathrm{RhB}^{+}$in TbTATAB vs. time curve fitted by the pseudosecondorder kinetic model. 


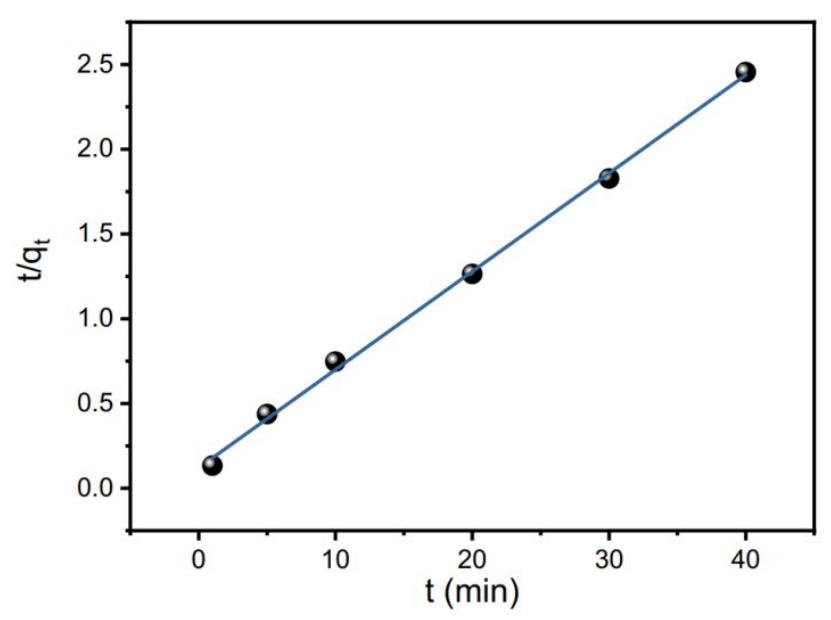

Figure S10. $t / q_{t}$ vs. time curve linear fitted by the pseudosecond-order kinetic model. $\mathrm{q}_{t}$ is the amount of $\mathrm{RhB}^{+}$adsorbed on the TbTATAB at time $\mathrm{t}(\mathrm{min})$.

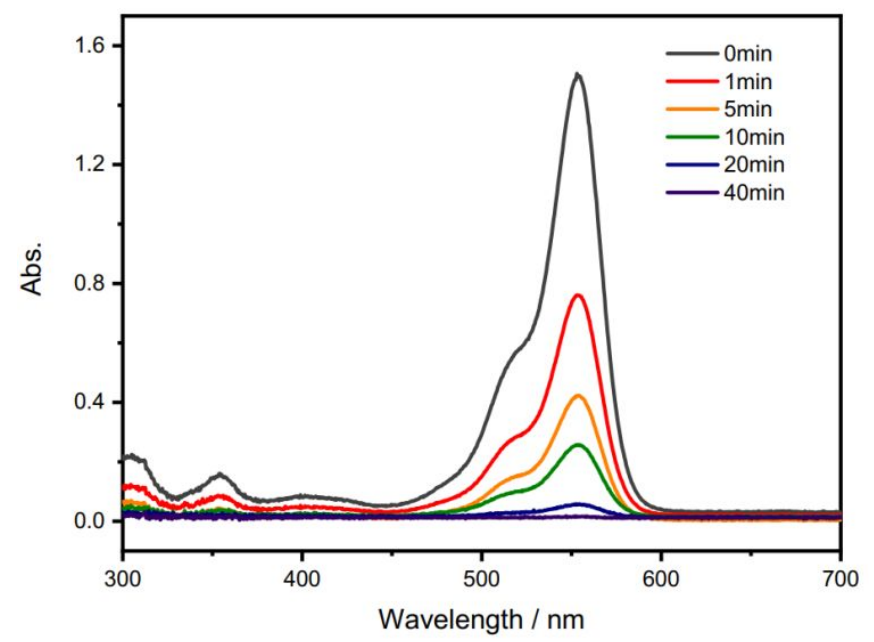

Figure S11. Temporal evolution of UV-Vis absorption spectra of $80 \mathrm{~mL} 0.1 \mathrm{mmol} / \mathrm{L} \mathrm{RhB}$ solution containing $200 \mathrm{mg}$ TbTATAB.

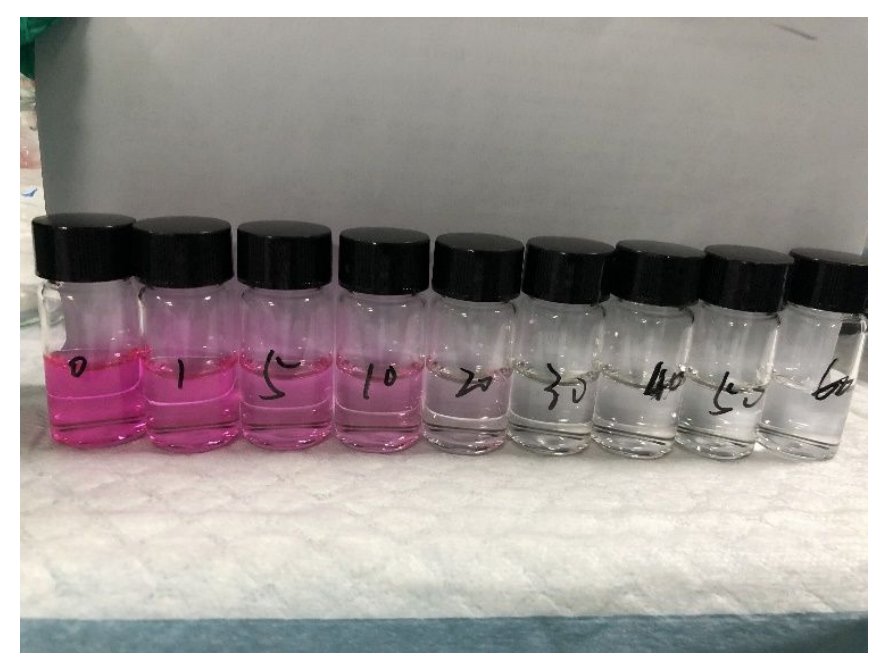

Figure S12. The photograph shows the changes in solution color with different adsorption times from $0 \mathrm{~min}$ to $60 \mathrm{~min}$. 


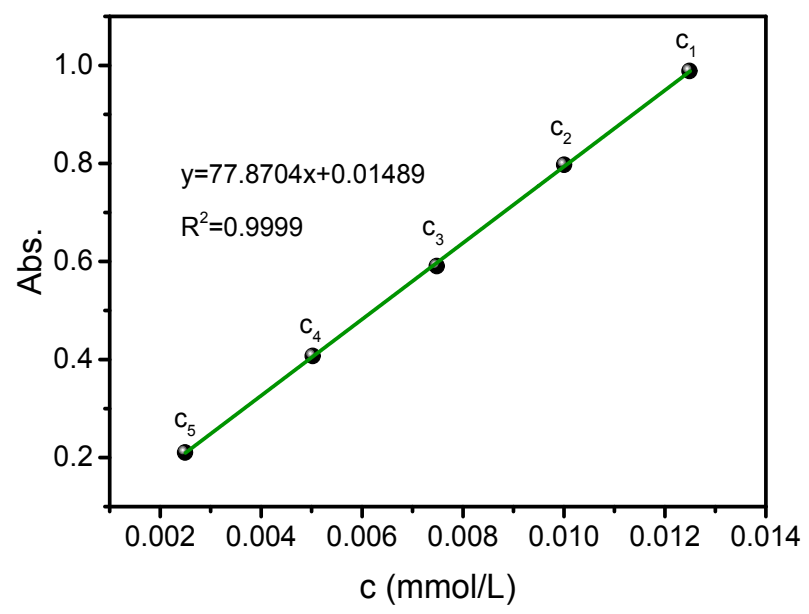

Figure S13. The linear fitted standard curve of UV-Vis absorption spectra at $553 \mathrm{~nm}$ from $\mathrm{c}_{1}$ $=0.0025 \mathrm{mmol} / \mathrm{L}$ to $c_{5}=0.0125 \mathrm{mmol} / \mathrm{L}\left(c_{2}=0.005 \mathrm{mmol} / \mathrm{L}, c_{3}=0.0075 \mathrm{mmol} / \mathrm{L}, c_{4}=0.01\right.$ $\mathrm{mmol} / \mathrm{L})$

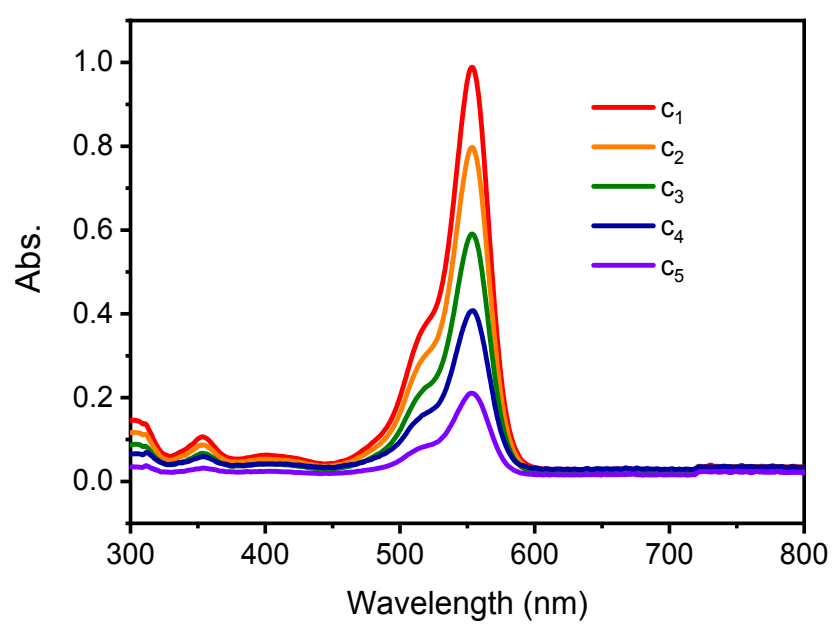

Figure S14. UV-Vis absorption spectra of $c_{1}$ to $c_{5}$ to fit the standard curve. 

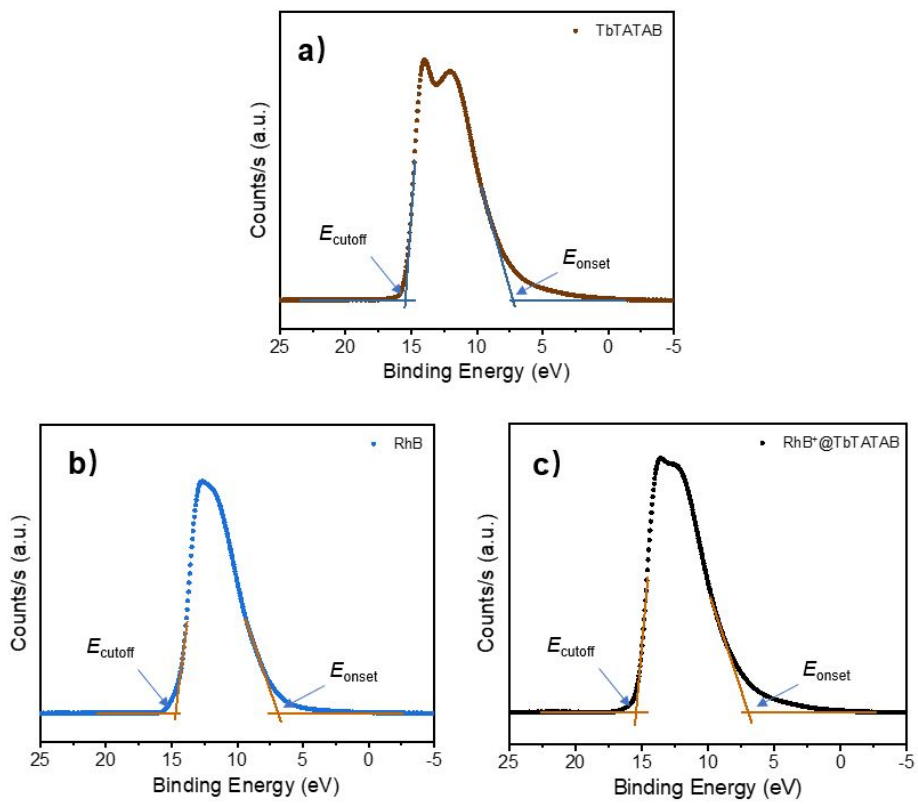

Figure S15. UPS spectra for different samples.

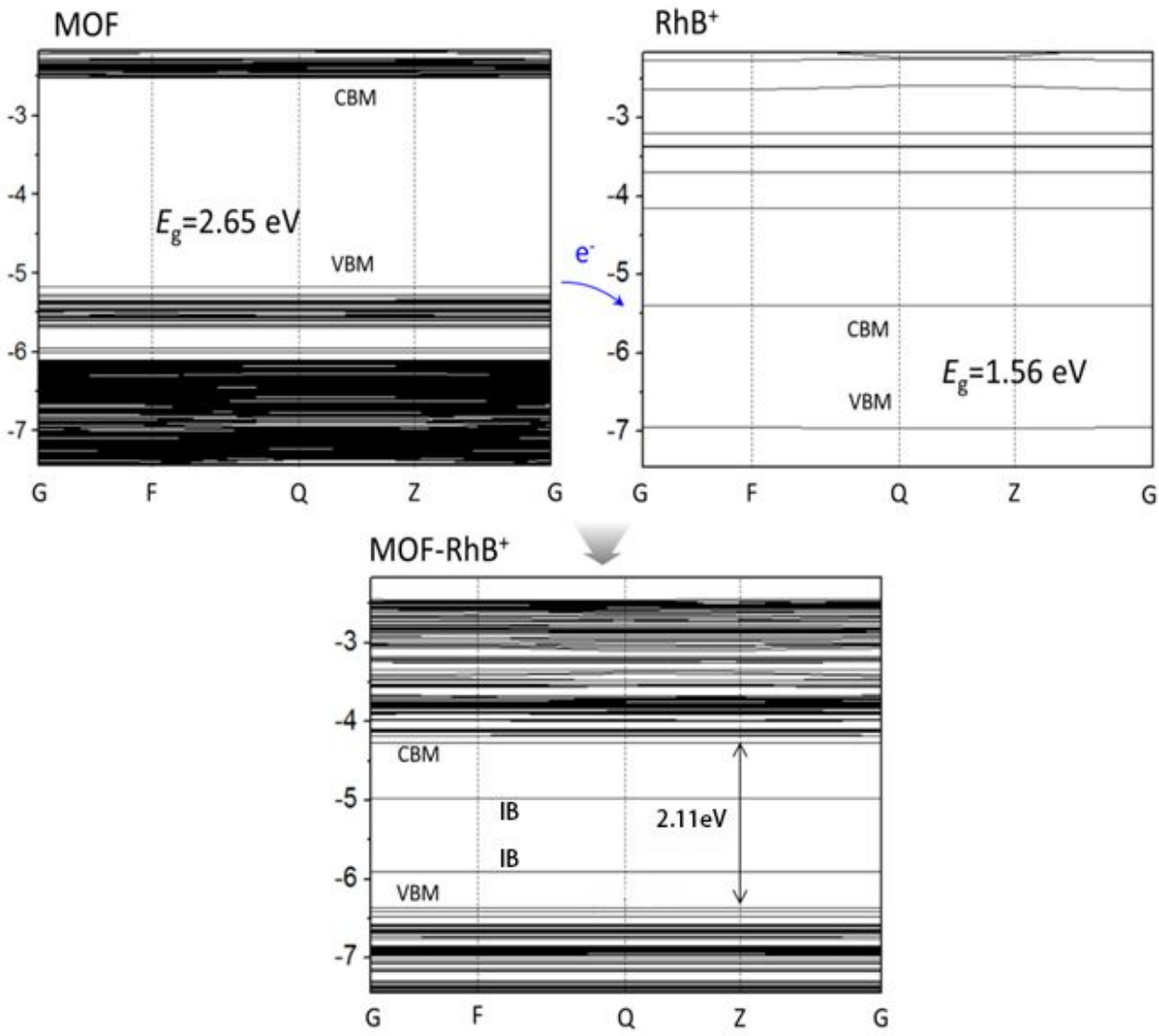

Figure S16. Band structures of TbTATAB, $\mathrm{RhB}^{+}$, and $\mathrm{RhB}^{+} @$ TbTATAB and the proposed mechanism of electron transfer from $\mathrm{MOF}$ to $\mathrm{RhB}^{+}$(denoted by the blue arrow). The calculated energy gap between the HOMO and LUMO $\left(\mathrm{E}_{\mathrm{g}}\right)$ of $\mathrm{RhB}^{+}(1.56 \mathrm{eV})$ is almost identical to the previously reported theoretical value $(1.557 \mathrm{eV}) .{ }^{5}$ After adsorbing $\mathrm{RhB}^{+}$, the intrinsic bandgap 
between the VBM and CBM of the host frameworks is decreased from $2.65 \mathrm{eV}$ to $2.11 \mathrm{eV}$.
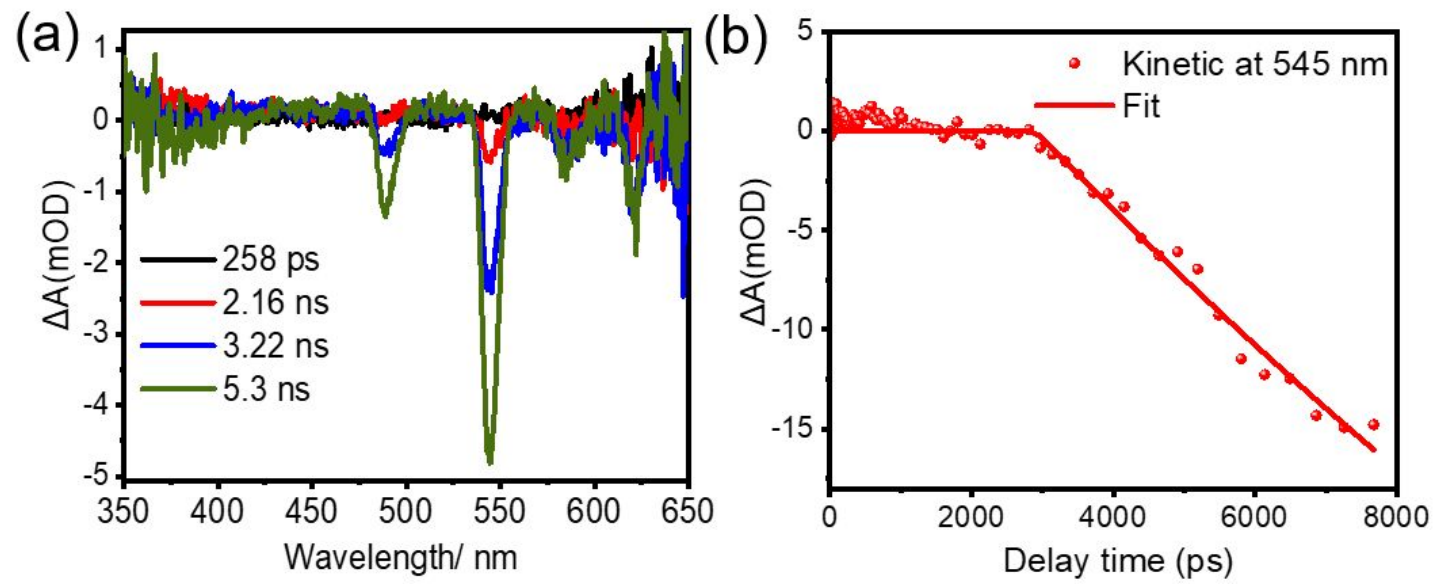

Figure S17. (a) Femtosecond transient absorption of TbTATAB excited by $300 \mathrm{~nm}(200 \mu \mathrm{W})$ and (b) the kinetics and fitting plot at $545 \mathrm{~nm}$.
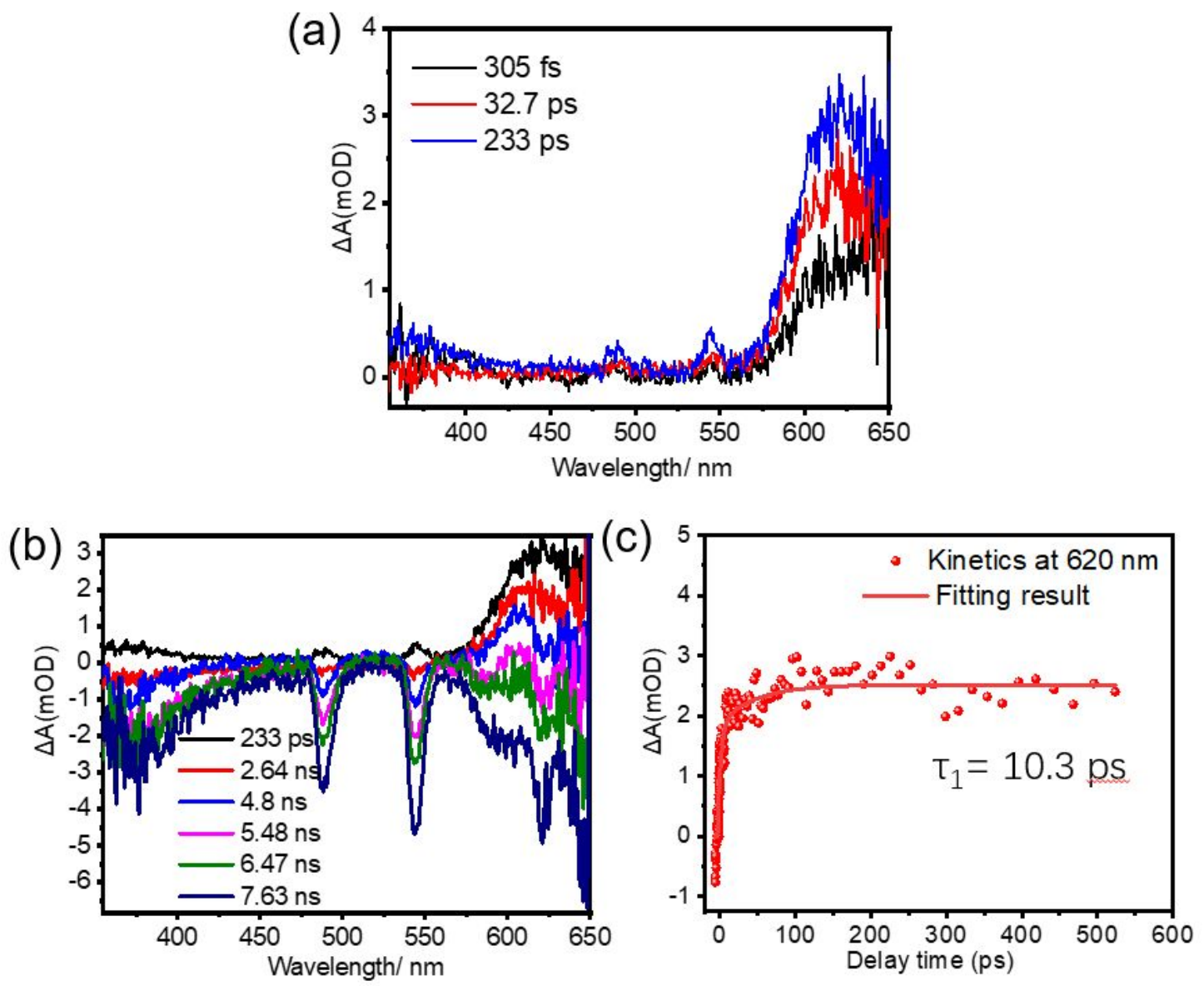

Figure S18. (a and b) Femtosecond transient absorption of RhB ${ }^{+} @$ TbTATAB excited by 300 $\mathrm{nm}(200 \mu \mathrm{W})$ and $(\mathrm{c})$ the kinetics and fitting plot at $620 \mathrm{~nm}$. 

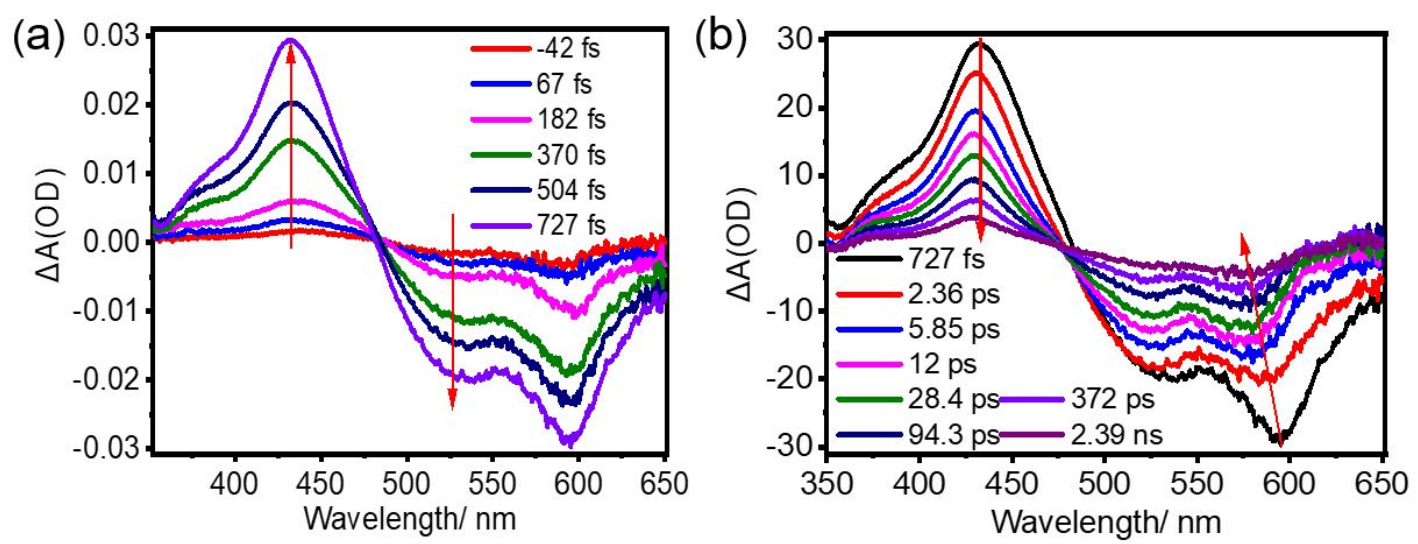

Figure S19. (a and b) Femtosecond transient absorption of RhB excited by $300 \mathrm{~nm}(200 \mu \mathrm{W})$.

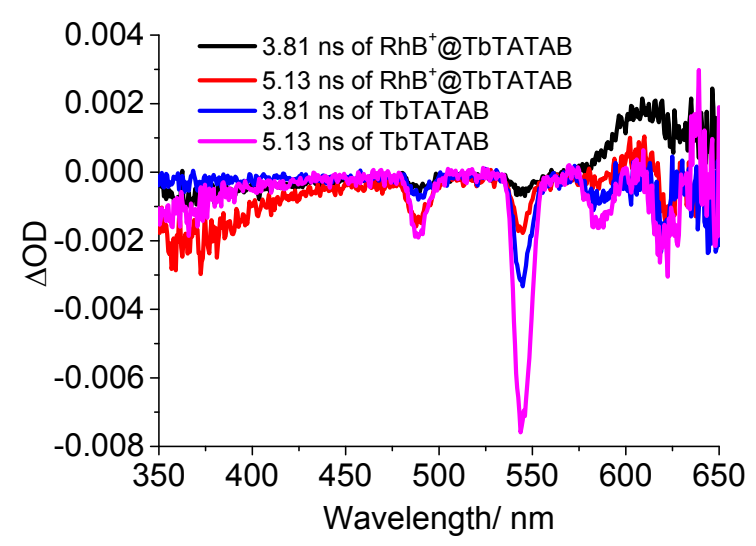

Figure S20. The comparison results on the transient absorption of TbTATAB and RhB $@$ TbTATAB at different delay times.
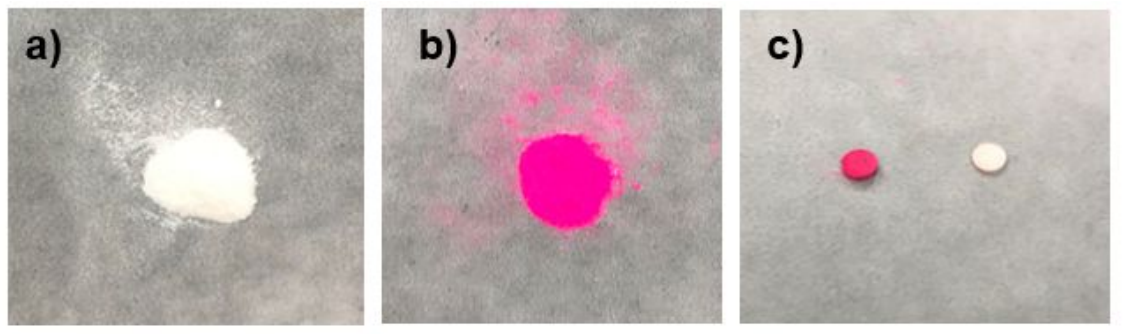

Figure S21. a) and b) Pictures of TbTATAB before and after RhB adsorption. c) Pelleted wafers for the electrical property test. 


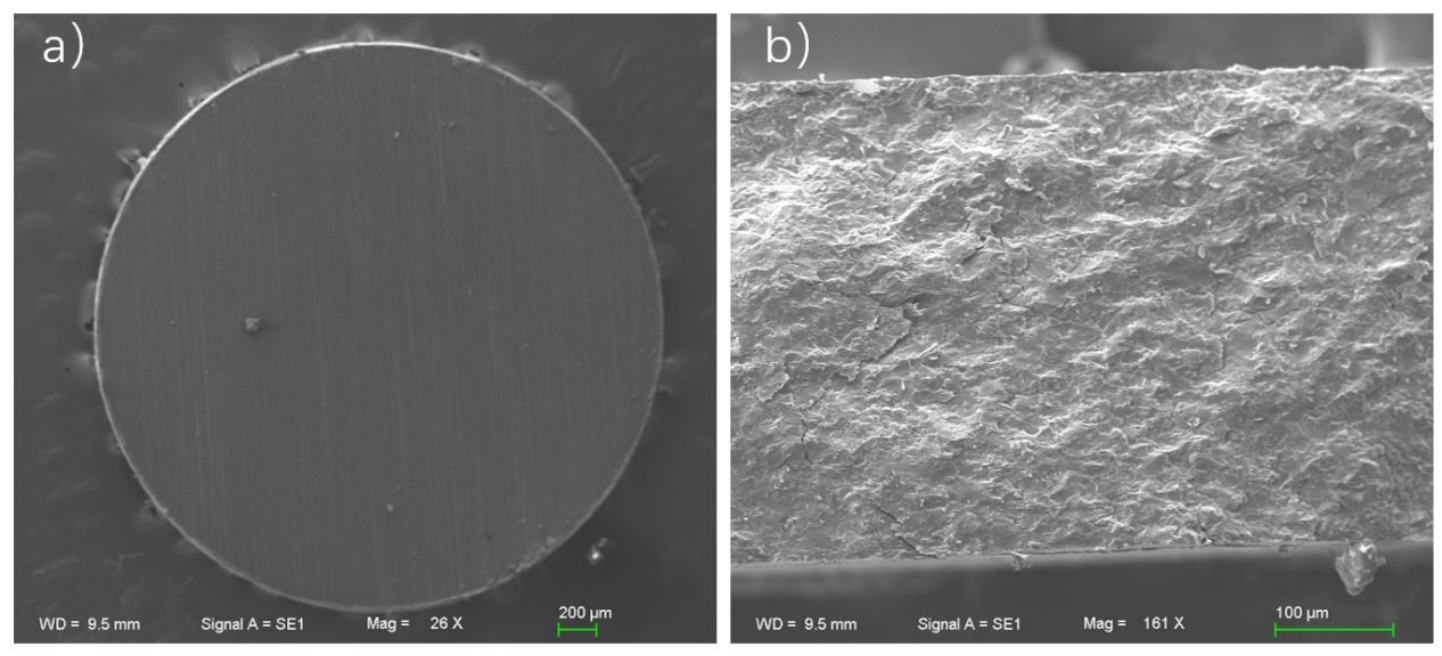

Figure S22. SEM image of a) the surface of pelleted $\mathrm{RhB}^{+} @$ TbTATAB and b) transverse section of pelleted $\mathrm{RhB}^{+} @$ TbTATAB.

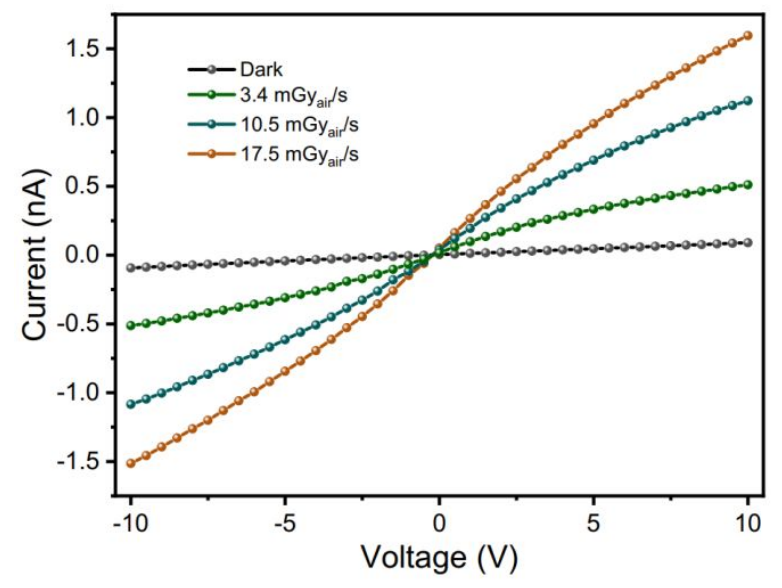

Figure S23. I-V curves for the RhB ${ }^{+} @$ TbTATAB-based detector under different X-ray dose rates.

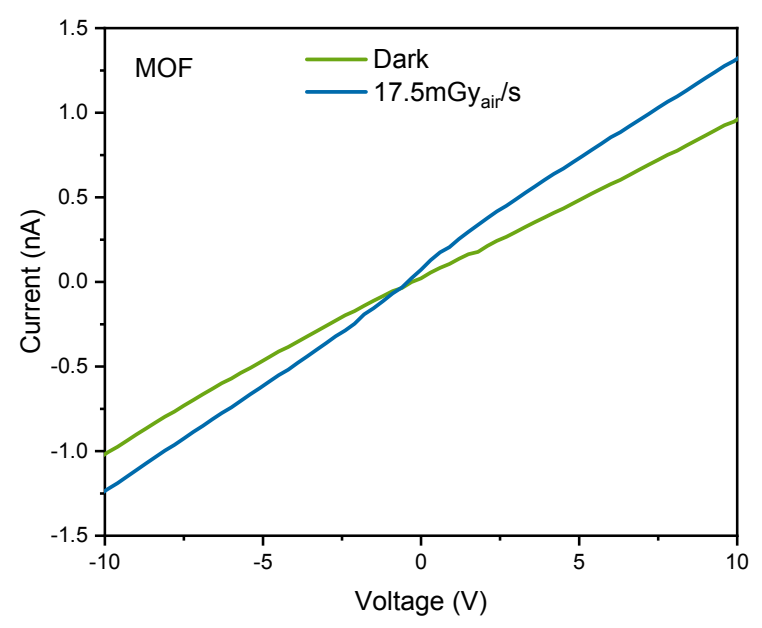

Figure S24. I-V curves for the TbTATAB-based detector under dark conditions and X-ray irradiation. 


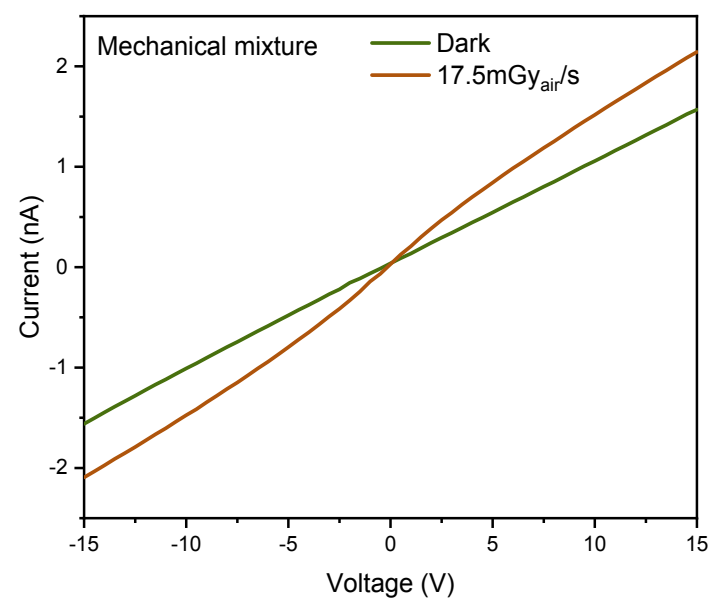

Figure S25. I-V curves for the mechanical mixture of the TbTATAB- and RhB-based detectors under dark conditions and X-ray irradiation.

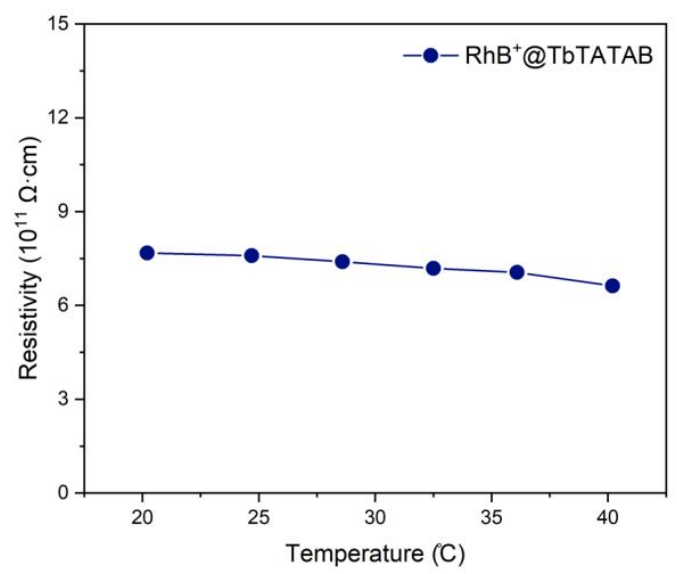

Figure S26. Temperature-dependent resistivity of RhB $@$ @TbTATAB.

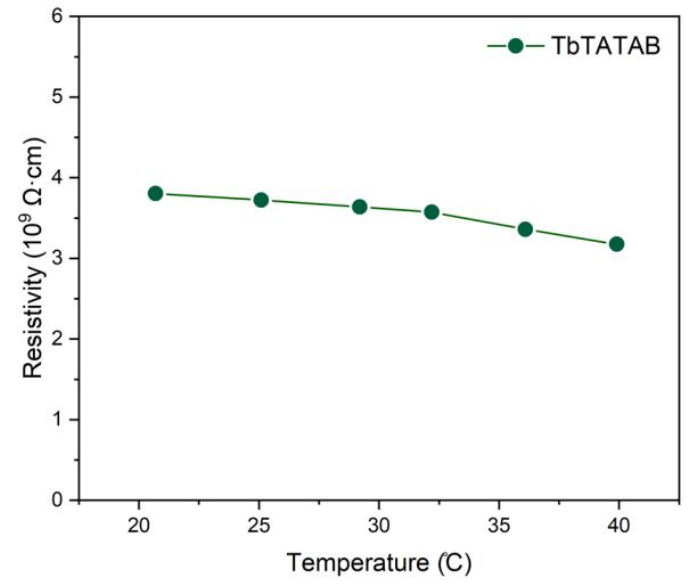

Figure S27. Temperature-dependent resistivity of TbTATAB. 


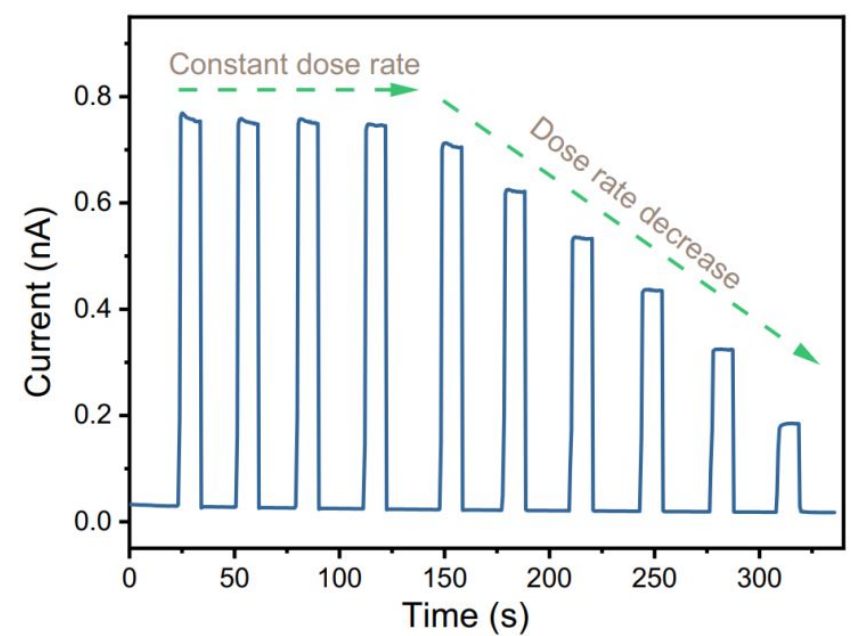

Figure S28. I-t curve for the $\mathrm{RhB}^{+} @$ TbTATAB-based detector under X-ray irradiation at different dose rates.

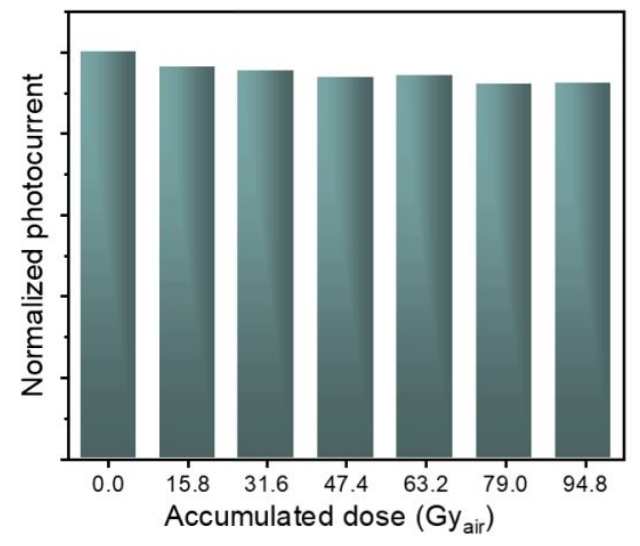

Figure S29. Normalized photocurrent of the $\mathrm{RhB}^{+} @$ TbTATAB-based detector under X-ray irradiation with different accumulated doses.

Table S2. Summary of the X-ray detection performances for selected materials.

\begin{tabular}{|c|c|c|c|}
\hline Material & $\begin{array}{c}\text { Sensitivity } \\
\left(\mu \mathrm{C} \mathrm{Gy} \mathrm{air}^{-1} \mathrm{~cm}^{-2}\right)\end{array}$ & $\begin{array}{c}\text { Mobility-lifetime } \\
\text { product } \\
\left(\mathrm{cm}^{2} \cdot \mathrm{V}^{-1}\right)\end{array}$ & Ref. \\
\hline RhB $^{+} @$ TbTATAB & 51.9 & $1.12 \times 10^{-3}$ & This work \\
\hline \multicolumn{4}{|c|}{ Perovskites based X-ray detectors } \\
\hline $\mathrm{Cs}_{2} \mathrm{AgBiBr}_{6} \mathrm{SC}$ & 105 & $3.75 \times 10^{-3}$ & Nat. Photon. 2017, 11, 726 \\
\hline$(\mathrm{BA})_{2} \mathrm{CsAgBiBr}_{7} \mathrm{SC}$ & 4.2 & $1.21 \times 10^{-3}$ & $\begin{array}{c}\text { Angew. Chem. Int. Ed. 2019, } \\
131,15904\end{array}$ \\
\hline $\mathrm{MAPbI}_{3}$ wafer & 2527 & $2 \times 10^{-4}$ & Nat. Photon. 2017, 11, 436 \\
\hline
\end{tabular}




\begin{tabular}{|c|c|c|c|}
\hline SCU-12 MOFs pellet & 23.8 & $1.3 \times 10^{-4}$ & $\begin{array}{c}\text { J. Am. Chem. Soc. } \mathbf{2 0 1 9}, \\
141,8030\end{array}$ \\
\hline Ni-DABDT flexible detector & 98.6 & $3.26 \times 10^{-4}$ & Nano Lett., 2021, 21, 6983 \\
\hline SCU-13 MOFs pellet & 4.54 & $3.76 \times 10^{-4}$ & $\begin{array}{c}\text { Angew. Chem. Int. Ed. } 2020 \text {, } \\
\text { 132, } 11954\end{array}$ \\
\hline $\mathrm{Cu}$-DABDT-MOFs & 78.7 & $6.49 \times 10^{-4}$ & $\begin{array}{l}\text { Chem. Commun. 2021, 57, } \\
8612\end{array}$ \\
\hline \multicolumn{4}{|c|}{ Commerically available X-ray detectors } \\
\hline$\alpha-\mathrm{Se}$ & 20 & & $\begin{array}{c}\text { J. Phys. D. Appl. Phys. 2000, } \\
33,2853\end{array}$ \\
\hline $\mathrm{Cd}_{0.9} \mathrm{Zn}_{0.1} \mathrm{Te}$ & $\approx 3$ & & Tech. Phys. 2010, 55, 306 \\
\hline
\end{tabular}

\section{References:}

(1) Zhang, N.-N.; Sa, R.-J.; Sun, S.-S.; Li, M.-D.; Wang, M.-S.; Guo, G.-C., Photoresponsive triazolebased donor-acceptor molecules: color change and heat/air-stable diradicals. J. Mater. Chem. C 2019, 7 , 3100-3104.

(2) Hess, B.; Kutzner, C.; van der Spoel, D.; Lindahl, E., Gromacs 4: Algorithms for Highly Efficient, Load-Balanced, and Scalable Molecular Simulation. J. Chem. Theory Comput. 2008, 4, 435-447.

(3) Wilmer, C. E.; Kim, K. C.; Snurr, R. Q., An Extended Charge Equilibration Method. J. Phys. Chem. Lett. 2012, 3, 2506-2511.

(4) Bussi, G.; Donadio, D.; Parrinello, M., Canonical Sampling through Velocity Rescaling. J. Chem. Phys. 2007, 126, 014101.

(5) Parrinello, M.; Rahman, A., Polymorphic Transitions in Alkali-Halides - a Molecular-Dynamics Study. J. Phys. 1981, 42, 511-515.

(6) Darden, T.; York, D.; Pedersen, L., Particle Mesh Ewald - an N.Log(N) Method for Ewald Sums in Large Systems. J. Chem. Phys. 1993, 98, 10089-10092.

(7) Hess, B.; Bekker, H.; Berendsen, H. J. C.; Fraaije, J. G. E. M., Lincs: A Linear Constraint Solver for Molecular Simulations. J. Comput. Chem. 1997, 18, 1463-1472.

(8) Clark, S. J.; Segall, M. D.; Pickard, C. J.; Hasnip, P. J.; Probert, M. J.; Refson, K.; Payne, M. C., First Principles Methods Using Castep. Z Kristallogr 2005, 220, 567-570.

(9) Perdew, J. P.; Chevary, J. A.; Vosko, S. H.; Jackson, K. A.; Pederson, M. R.; Singh, D. J.; Fiolhais, C., Erratum: Atoms, Molecules, Solids, and Surfaces: Applications of the Generalized Gradient Approximation for Exchange and Correlation. Phys Rev B Condens Matter 1993, 48, 4978.

(10) Perdew, J. P.; Chevary, J. A.; Vosko, S. H.; Jackson, K. A.; Pederson, M. R.; Singh, D. J.; Fiolhais, C., Atoms, Molecules, Solids, and Surfaces: Applications of the Generalized Gradient Approximation for Exchange and Correlation. Phys Rev B Condens Matter 1992, 46, 6671-6687.

(11) Perdew, J. P.; Burke, K.; Wang, Y., Generalized Gradient Approximation for the Exchange- 
Correlation Hole of a Many-Electron System. Phys Rev B Condens Matter 1996, 54, 16533-16539.

(12) Henkelman, G.; Arnaldsson, A.; Jonsson, H., A Fast and Robust Algorithm for Bader Decomposition of Charge Density. Comp Mater Sci 2006, 36, 354-360.

(13) M. J. Frisch, G. W. T., H. B. Schlegel, G. E. Scuseria, M. A. Robb, J. R. Cheeseman, G. Scalmani, V. Barone, G. A. Petersson, H. Nakatsuji, X. Li, M. Caricato, A. Marenich, J. Bloino, B. G. Janesko, R. Gomperts, B. Mennucci, H. P. Hratchian, J. V. Ortiz, A. F. Izmaylov, J. L. Sonnenberg, D. WilliamsYoung, F. Ding, F. Lipparini, F. Egidi, J. Goings, B. Peng, A. Petrone, T. Henderson, D. Ranasinghe, V. G. Zakrzewski, J. Gao, N. Rega, G. Zheng, W. Liang, M. Hada, M. Ehara, K. Toyota, R. Fukuda, J. Hasegawa, M. Ishida, T. Nakajima, Y. Honda, O. Kitao, H. Nakai, T. Vreven, K. Throssell, J. A. Montgomery, Jr., J. E. Peralta, F. Ogliaro, M. Bearpark, J. J. Heyd, E. Brothers, K. N. Kudin, V. N. Staroverov, T. Keith, R. Kobayashi, J. Normand, K. Raghavachari, A. Rendell, J. C. Burant, S. S. Iyengar, J. Tomasi, M. Cossi, J. M. Millam, M. Klene, C. Adamo, R. Cammi, J. W. Ochterski, R. L. Martin, K. Morokuma, O. Farkas, J. B. Foresman, and D. J. Fox, Gaussian 09, Rev. E.01, 2009.

(14) Zhao, Y.; Truhlar, D. G., The M06 Suite of Density Functionals for Main Group Thermochemistry, Thermochemical Kinetics, Noncovalent Interactions, Excited States, and Transition Elements: Two New Functionals and Systematic Testing of Four M06-Class Functionals and 12 Other Functionals. Theor. Chem. Acc. 2008, 120, 215-241.

(15) Hehre, W. J.; Ditchfield, R.; Pople, J. A., Self-Consistent Molecular Orbital Methods. Xii. Further Extensions of Gaussian-Type Basis Sets for Use in Molecular Orbital Studies of Organic Molecules. The Journal of Chemical Physics 1972, 56, 2257-2261.

(16) Grimme, S.; Antony, J.; Ehrlich, S.; Krieg, H., A Consistent and Accurate Ab Initio Parametrization of Density Functional Dispersion Correction (Dft-D) for the 94 Elements H-Pu. The Journal of chemical physics 2010, 132, 154104. 